\title{
Axial Anomaly Effect in Chiral p-wave Superconductor
}

\author{
Jun Goryo* \\ Yukawa Institute for Theoretical Physics, Kyoto University, Kyoto, 606-8502 Japan.
}

\begin{abstract}
We analyze the chiral $p$-wave superconductor in the low temperature region. The superconductor has a $\varepsilon_{x} p_{x}+i \varepsilon_{y} p_{y}$-wave gap in two-dimensional space (2D). Near the second superconducting transition point, the system could be described by a quasi-1D chiral $p$-wave model in $2 \mathrm{D}$. The axial anomaly occurs in such a model and causes an accumulation of the quasiparticle in an inhomogeneous magnetic field. The effect is related to the winding number of the gap.
\end{abstract}

*present address; Inst. Solid State Phys., U. Tokyo, Kashiwanoha, Kashiwa 277-0882, Japan. e-mail; jfg@issp.u-tokyo.ac.jp 
Chiral $p$-wave superfluidity is realized in the superfluid ${ }^{3} \mathrm{He}-\mathrm{A}$ [1]. Recently, the possibility of chiral $p$-wave superconductivity has been argued in $\mathrm{Sr}_{2} \mathrm{RuO}_{4}$ [2]. In such superfluids or superconductors, the condensation of the Cooper pairs, which have orbital angular momentum along the same direction, occurs. Therefore, time-reversal symmetry (T) and also parity $(\mathrm{P})$ in two-dimensional space (2D) are violated. We investigate an axial anomaly effect in the chiral $p$-wave superconductor near the second superconducting transition point [3]. It is revealed that the axial anomaly causes $\mathrm{P}$ - and T-violating phenomena related to the topologically quantized number at sufficiently low temperatures.

The axial anomaly was originally pointed out in the Dirac QED in 3D [6 6]. It is the phenomenon in which a symmetry under the phase transformation $e^{i \gamma_{5} \alpha}$ of the Dirac field in the action at the classical level is broken in the quantum theory. Here, $\alpha$ is a constant and $\gamma_{5}$ is a hermitian matrix which anti-commutes with all of the Dirac matrices $\gamma_{\mu}$, where $\mu$ is the spacetime index. The Adler-Bardeen's theorem guarantees the absence of higher order corrections to the divergence of the axial current [4.5]. Therefore, the exact calculation of the two-photon decay rate of the neutral $\pi$ meson can be carried out. It has been pointed out that the same results are obtained by using the path-integral formalism and the relation between the axial anomaly and topological quantized numbers has been clarified through the Atiyah-Singer index theorem [0] 9].

It has been pointed out that the axial anomaly also plays an important role in the quantum Hall effect (QHE) in the 2D massive Dirac QED. In 2D, the mass term of the Dirac fermion violates $\mathrm{P}$ and $\mathrm{T}$ similar to the magnetic field, and the Hall effect may occur. It was shown that the existence of the Hall current and its quantization are caused by the axial anomaly in 1D [10 [13].

The relation between the axial anomaly and QHE in 2D electron gas in the magnetic field was also discussed, and the quantized Hall conductance is expressed by the winding number of the fermionic propagator in the momentum space [12 [16]. Other applications of the axial anomaly to condensed matter physics has been studied in the field of the superfluid ${ }^{3} \mathrm{He}$ in 3D [1, 17, 18], and also in some 1D systems [19,20]. 
The phenomena caused by the axial anomaly are related to the topologically quantized numbers. On the analogy of QHE, it is expected that the axial anomaly also plays an important role in other $\mathrm{P}$ - and $\mathrm{T}$-violating $2 \mathrm{D}$ systems. In this letter, we show an axial anomaly effect in a chiral $p$-wave superconductor in $2 \mathrm{D}$, which has the spin-triplet $\varepsilon_{x} p_{x}+i \varepsilon_{y} p_{y}$-wave symmetry. $\mathrm{P}$ and T-violation occur whenever both $\varepsilon_{x}$ and $\varepsilon_{y}$ are nonzero. We show that near the second superconducting phase transition (2ndSCPT) point the axial anomaly in 1D causes an accumulation of the mass density of the quasiparticle in an inhomogeneous magnetic field. The effect is related to the winding number of the gap; $\operatorname{sgn}\left(\varepsilon_{x} \varepsilon_{y}\right)$ [21,22]. We use the $2+1$-dimensional Euclidian spacetime and the natural unit $(\hbar=c=1)$ in the present paper.

Let us consider 2ndSCPT in the chiral $p$-wave state. It has been proposed theoretically that such a transition occurs under uniaxial pressure in the $x-y$ plane (the basal plane) [3]. 2ndSCPT caused by an exact in-plane magnetic field has also been argued [23], but our argument is not applicable to this transition because the anomaly effect which we will show requires a magnetic field perpendicular to the basal plane. The orbital dependence of the gap near the transition point is shown in Fig. 1. For simplicity, we assume a circular fermi surface in the normal state. There are two tiny gap points around $\mathbf{p}=\left( \pm p_{\mathrm{F}}, 0\right)$, where $p_{\mathrm{F}}$ is the Fermi momentum. Therefore, the quasiparticle excitations along the $x$-axis become dominant in the low temperature region. We can describe such a situation effectively by using a quasi-1D model. For the present, we consider the superfluid just for simplicity, and will extend our argument to the supercondcuting case by taking into account the Meissner effect. We assume a linearized fermion spectrum and a spin-triplet chiral $p$-wave gap near the Fermi surface in the normal state (i.e. $p_{x}= \pm p_{\mathrm{F}}+\delta p_{x}, \delta p_{x}<<p_{\mathrm{F}}$ ) written as,

$$
\begin{aligned}
\epsilon_{\mathrm{R}, \mathrm{L}}(\mathbf{p}) & = \pm v_{\mathrm{F}} \delta p_{x} \\
\Delta(\mathbf{p}) & =i \sigma_{3} \sigma_{2} \frac{\Delta}{p_{\mathrm{F}}}\left(\varepsilon_{x} p_{x}+i \varepsilon_{y} p_{y}\right) \\
& \simeq i \sigma_{3} \sigma_{2} \Delta\left( \pm \varepsilon_{x}+i \varepsilon_{y} \frac{p_{y}}{p_{\mathrm{F}}}\right)
\end{aligned}
$$

where $v_{\mathrm{F}}$ is the Fermi velocity. $\epsilon_{R}(\mathbf{p})\left(\epsilon_{L}(\mathbf{p})\right)$ is the kinetic energy for the right (left) mover. 
$\varepsilon_{x}$ and $\varepsilon_{y}$ are parameters and presently they satisfy $\varepsilon_{x}<<1$ and $\varepsilon_{y} \sim 1$.

The Lagrangian of our model is written as

$$
\begin{aligned}
\mathcal{L}= & \bar{\Psi}_{\mathbf{p}}\left[\left\{i \partial_{\tau}+\mu \frac{d \mathrm{~B}_{z}}{d y} y \sigma_{3}\right\} \otimes \gamma_{\tau}\right. \\
& \left.+v_{\mathrm{F}} \delta p_{x} \hat{1} \otimes \gamma_{x}+\frac{\Delta}{p_{\mathrm{F}}} \varepsilon_{y} p_{y} \sigma_{3} \otimes \gamma_{y}-i \Delta \varepsilon_{x} \sigma_{3} \otimes \hat{1}\right] \Psi_{\mathbf{p}}
\end{aligned}
$$

Here, we use the Bogoliubov-Nambu representation with an isospin $\alpha=1,2$,

$$
\Psi(\mathbf{x})=e^{i p_{\mathrm{F}} x}\left(\begin{array}{c}
\psi_{\mathrm{R}}(\mathbf{x}) \\
i \sigma_{2} \psi_{\mathrm{L}}^{*}(\mathbf{x})
\end{array}\right)
$$

and $\Psi_{\mathbf{p}}$ is its Fourier transform. $\psi_{\mathrm{R}}(\mathbf{x})$ and $\psi_{\mathrm{L}}(\mathbf{x})$ are the slowly varying fields for the right mover and the left mover with a real spin index $s=1,2$, respectively. The matrices $\gamma_{\tau}=$ $\tau_{1}, \gamma_{x}=\tau_{2}$ and $\gamma_{y}=\tau_{3}$ are the $2 \times 2$ Pauli matrices with isospin indices and $\sigma_{i}(i=1,2,3)$ are the $2 \times 2$ Pauli matrices with spin indices. The symbol $\sigma_{i} \otimes \gamma_{\tau, x, y}$ represents the direct product. $\bar{\Psi}$ is defined as $\bar{\Psi}=-i \Psi^{\dagger} \gamma_{\tau}$. We assume a magnetic field, which is directed to the $z$-axis (the $c$-axis in the crystal) and has a constant gradient in the $y$-direction, i.e., $B_{z}(y)=\left(d B_{z} / d y\right) y$, and $\left(d B_{z} / d y\right)=$ const. Because we are considering the superfluid for the present, the magnetic field couples with the fermion only through the Zeeman term $\mu B_{z} \bar{\Psi} \sigma_{3} \otimes \gamma_{\tau} \Psi$, where $\mu$ is the magnetic moment of the fermion. In general, the magnitude of the gap $\Delta$ depends on the magnetic field, but we neglect this fact because it has become apparent that our result does not depend on $\Delta$.

We note that the Lagrangian is equivalent to that of the 2D Dirac QED [24] in a constant electric field, except for the existence of $\sigma_{3}$. It has been shown that the axial anomaly causes the quantized Hall effect in the 2D Dirac system [10 13]. In our model, the physical value which corresponds to the quantized Hall current $j_{x}=e\left\langle\bar{\Psi}_{\mathrm{D}} \gamma_{x} \Psi_{\mathrm{D}}\right\rangle$ in 2D Dirac theory $\left(\Psi_{\mathrm{D}}\right.$ is the Dirac field and $\langle\cdots\rangle$ is the expectation value for the ground state) is the mass (charge) density, because $e\left\langle\bar{\Psi} \gamma_{x} \Psi\right\rangle=e\left\langle\psi_{\mathrm{R}}^{\dagger} \psi_{\mathrm{R}}+\psi_{\mathrm{L}}^{\dagger} \psi_{\mathrm{L}}\right\rangle=e\left\langle\rho_{e}\right\rangle$, where $e$ represents a mass of the quasiparticle, and represents an electric charge when we consider a superconductor. The reason why $e\left\langle\bar{\Psi} \gamma_{x} \Psi\right\rangle$ becomes the charge density, not the current, is the remarkable 
difference between $\Psi_{\mathrm{D}}$ and $\Psi . \Psi_{\mathrm{D}}$ is a two-component fermion field and each component has the same charge. $\Psi$ is also two component (two isospin) but the signs of their charges are opposite.

Let us calculate the expectation value of the mass (charge) density

$$
\left\langle\rho_{e}(\mathbf{x})\right\rangle=\operatorname{Tr}\left[\frac{e \sigma_{3} \gamma_{x}}{i \not d \gamma_{x}+v_{\mathrm{F}} \delta p_{x} \sigma_{3} \otimes \gamma_{x}-i \Delta \varepsilon_{x}}\right] .
$$

A hermitian operator $\not d$ in 1D (in the $y$-direction) is defined as

$$
\not d=\left\{i \partial_{\tau} \sigma_{3}+\mu \frac{d B_{z}}{d y} y \hat{1}\right\} \otimes \tilde{\gamma}_{\tau}+\frac{\Delta}{p_{\mathrm{F}}} \varepsilon_{y} p_{y} \hat{1} \otimes \tilde{\gamma}_{y},
$$

where $\tilde{\gamma}_{\tau}=-\gamma_{y}=-\tau_{3}$ and $\tilde{\gamma}_{y}=\gamma_{\tau}=\tau_{1}$. We define $\gamma_{5}$ as $\gamma_{5}=i \tilde{\gamma}_{\tau} \tilde{\gamma}_{y}=-\gamma_{x}=-\tau_{2}$, and it is anti-commute with $\tilde{\gamma}_{\tau}$ and $\tilde{\gamma}_{y}$, therefore, $\gamma_{5}$ is a hermitian matrix which satisfies $\left\{\gamma_{5}, \not d\right\}=0$. These findings suggest that if an eigenstate $u_{n}$ of $\not d$ with a nonzero eigenvalue $\xi_{n}(0<n)$ exists (i.e. $\left.\not d u_{n}=\xi_{n} u_{n}\right), \gamma_{5} u_{n}$ should be another eigenstate with an eigenvalue $-\xi_{n}$. If zero modes of $\not d$ exist (i.e. $\not d u_{0}=0$ and $\left.\not d \gamma_{5} u_{0}=0\right)$, they are divided into two groups. One of them is $u_{0}^{(+)}=(1 / 2)\left(1+\gamma_{5}\right) u_{0}$ with an eigenvalue $\gamma_{5}=+1$ and the other is $u_{0}^{(-)}=(1 / 2)\left(1-\gamma_{5}\right) u_{0}$ with an eigenvalue $\gamma_{5}=-1$, since $\gamma_{5}^{2}=1$.

Let us research the eigenmodes of $\not$. The expectation value of $\phi^{2}$ is

$$
\begin{aligned}
\left(u_{n}, \phi^{2} u_{n}\right) & =\left|\omega_{c}\right|\left(n+\frac{1}{2}\right)+\frac{\omega_{c}}{2}\left(u_{n}, \gamma_{5} u_{n}\right), \\
\omega_{c} & =\mu \frac{d B_{z}}{d y} \frac{2 \Delta}{p_{\mathrm{F}}} \varepsilon_{y},
\end{aligned}
$$

where $u_{n}=u_{n}\left(y-y_{c}\left(p_{\tau}, \sigma_{3}\right)\right)$ is the eigenfunction of the harmonic oscillator with the frequency $\omega_{c}$. The oscillator is centered at $y_{c}\left(p_{\tau}, \sigma_{3}\right)=-\left(d B_{z} / d y\right)^{-1}\left(p_{\tau} / \mu\right) \sigma_{3}$. Equation (6) indicates that only zero modes which belong to $u_{0}^{-}\left(u_{0}^{+}\right)$exist when $0<\omega_{c}\left(\omega_{c}<0\right)$. This suggests the non-conservation of the vacuum expectation value of the axial charge which is defined in the second-quantized formalism as

$$
\begin{aligned}
\left\langle Q_{5}\right\rangle & =\left\langle N_{+}-N_{-}\right\rangle, \\
N_{ \pm} & =\int d p_{y} \hat{u}_{0}^{\dagger( \pm)} \hat{u}_{0}^{( \pm)},
\end{aligned}
$$


while the classical 1D theory $\mathcal{L}_{1 \mathrm{D}}=\bar{\Psi} \not d \Psi$ has the axial symmetry $\Psi \rightarrow e^{i \alpha \gamma_{5}} \Psi$, i.e., the axial anomaly occurs. Here, $\hat{u}_{0}^{ \pm}$is a second-quantized fermionic field. The anomaly comes from the spectral asymmetry of zero modes, as in the discussions presented in Refs. [10 [13]25]. In the free system, the energy spectrum of $u_{0}^{( \pm)}$is $p_{0}= \pm \frac{\Delta}{p_{\mathrm{F}}} \varepsilon_{y} p_{y}$ for spin down $\left(\sigma_{3}=+1\right)$ states in Minkowski spacetime, and all of the negative energy states are filled while all of the positive energy states are empty and $\left\langle Q_{5}\right\rangle=0$. After we turn on the magnetic field adiabatically (for the present, we assume $0<\omega_{c}$ ), the energy spectrum of $u_{0}^{(+)}$is lowered and $\left\langle N_{+}\right\rangle$decreases (i.e., empty negative energy states arise on the spectrum of $u_{0}^{(+)}$); on the other hand, the energy spectrum of $u_{0}^{(-)}$is lifted and $\left\langle N_{-}\right\rangle$increases (i.e., filled positive energy states arise on the spectrum of $\left.u_{0}^{(-)}\right)$, and therefore, $\left\langle Q_{5}\right\rangle$ does not conserve. Finally $\left\langle N_{+}\right\rangle=0$ and only $u_{0}^{(-)}$exists. We see that the same argument is also valid for spin down $\left(\sigma_{3}=-1\right)$ states. The nonzero eigenvalues of $\phi^{2}$ are $E_{n}=\omega_{c}(n+1 / 2)$, since the inner product $\left(u_{n}, \gamma_{5} u_{n}\right)$ vanishes whenever $\phi u_{n} \neq 0$ because of the orthogonal relation between the eigenfunctions of the hermitian operator.

Next, we consider the eigenvalue problem of a 2D operator:

$$
D=\not d-\Delta \varepsilon_{x} \hat{1} \otimes \gamma_{x}=\not d+\Delta \varepsilon_{x} \hat{1} \otimes \gamma_{5} .
$$

Let

$$
\varphi_{n}=\left(\alpha_{n} u_{n}+\beta_{n} \gamma_{5} u_{n}\right) e^{i p_{x} x}
$$

stand for an eigenfunction. We use a representation for the $n$-th level such as

$$
\not d=\left(\begin{array}{cc}
\xi_{n} & 0 \\
0 & -\xi_{n}
\end{array}\right), u_{n}=\left(\begin{array}{l}
1 \\
0
\end{array}\right), \gamma_{5}=\left(\begin{array}{ll}
0 & 1 \\
1 & 0
\end{array}\right),
$$

where,

$$
\xi_{n}=\left\{\begin{array}{cl}
\sqrt{\left|\omega_{c}\right|\left(n+\frac{1}{2}\right)} & (n=1,2, \cdots), \\
0 & (n=0) .
\end{array}\right.
$$

Therefore, the eigenvalue equation is written as 


$$
\left(\begin{array}{cc}
\xi_{n} & \Delta \varepsilon_{x} \\
\Delta \varepsilon_{x} & -\xi_{n}
\end{array}\right)\left(\begin{array}{c}
\alpha_{n} \\
\beta_{n}
\end{array}\right)=\zeta_{n}\left(\begin{array}{c}
\alpha_{n} \\
\beta_{n}
\end{array}\right) .
$$

There are two eigenstates for an oscillator in the $n(\neq 0)$-th level written as

$$
\begin{aligned}
& \zeta_{n}^{( \pm)}\left(p_{x}\right)= \pm \sqrt{\xi_{n}^{2}+\Delta^{2} \varepsilon_{x}^{2}} \\
& \left(\begin{array}{c}
\alpha_{n}^{+} \\
\beta_{n}^{+}
\end{array}\right)=\frac{1}{C_{+}}\left(\begin{array}{c}
\zeta_{n}^{(+)}+\xi_{n} \\
\Delta \varepsilon_{x}
\end{array}\right), \\
& \left(\begin{array}{c}
\alpha_{n}^{-} \\
\beta_{n}^{-}
\end{array}\right)=\frac{1}{C_{-}}\left(\begin{array}{c}
-\Delta \varepsilon_{x} \\
-\zeta_{n}^{(-)}+\xi_{n}
\end{array}\right),
\end{aligned}
$$

where $C_{ \pm}$are normalization constants, but for $n=0$, there is only one eigenstate

$$
\begin{aligned}
\zeta_{0}\left(p_{x}\right) & =\frac{\omega_{c}}{\left|\omega_{c}\right|} \Delta\left|\varepsilon_{x}\right|, \\
\left(\begin{array}{c}
\alpha_{0} \\
\beta_{0}
\end{array}\right) & =\frac{1}{2}\left(\begin{array}{c}
1 \\
-\omega_{c} /\left|\omega_{c}\right|
\end{array}\right),
\end{aligned}
$$

because the solution should satisfy $\gamma_{5} \varphi_{0}=-\left(\omega_{c} /\left|\omega_{c}\right|\right) \varphi_{0}$. This condition comes from the axial anomaly in the $y$-direction.

Finally, we show the accumulation of the mass density from Eq. (4), which is derived as

$$
\begin{aligned}
\left\langle\rho_{e}(\mathbf{x})\right\rangle= & \operatorname{Tr}\left[\frac{-i e \sigma_{3}}{p-i v_{\mathrm{F}} \delta p_{x} \sigma_{3} \otimes \hat{1}}\right] \\
= & \sum_{n} \int_{-\infty}^{\infty} \frac{d p_{\tau}}{2 \pi} \int_{-\Lambda}^{\Lambda} \frac{d \delta p_{x}}{2 \pi} \operatorname{tr}\left[\frac{-i e \sigma_{3}\left|u_{n}\left(y-y_{0}\left(p_{\tau}, \sigma_{3}\right)\right)\right|^{2}}{\zeta_{n}\left(p_{x}\right)-i v_{\mathrm{F}} \delta p_{x} \sigma_{3}}\right] \\
= & \frac{e \mu}{2 \pi} \frac{d B_{z}}{d y} \sum_{n \neq 0} \int_{-\Lambda}^{\Lambda} \frac{d \delta p_{x}}{2 \pi} \operatorname{tr}\left[\frac{1}{\zeta_{n}^{(+)}\left(p_{x}\right)-i v_{\mathrm{F}} \delta p_{x} \sigma_{3}}+\frac{1}{\zeta_{n}^{(-)}\left(p_{x}\right)-i v_{\mathrm{F}} \delta p_{x} \sigma_{3}}\right] \\
& +\frac{e \mu}{2 \pi} \frac{d B_{z}}{d y} \int_{-\Lambda}^{\Lambda} \frac{d \delta p_{x}}{2 \pi} \operatorname{tr}\left[\frac{1}{\zeta_{0}\left(p_{x}\right)-i v_{\mathrm{F}} \delta p_{x} \sigma_{3}}\right] \\
= & \operatorname{sgn}\left(\varepsilon_{x} \varepsilon_{y}\right) e \mu N_{1 \mathrm{D}}(0) \frac{d B_{z}}{d y}
\end{aligned}
$$

where the symbol $t r$ represents a trace on the real spin, and we use the normal-orthogonal relation $\int d y\left|u_{n}\right|^{2}=1 . \Lambda$ is a momentum cutoff and we assume a relation $|\Delta|<<\Lambda^{2} / 2 m<<$ $\epsilon_{F} \cdot N_{1 \mathrm{D}}(0)=\left(2 \pi v_{\mathrm{F}}\right)^{-1}$ is the density of state at the Fermi surface in 1D. All of the $n \neq 0$ 
parts are canceled out because of the co-existence of the eigenvalues $\zeta_{n}^{(+)}$and $\zeta_{n}^{(-)}$. Only the $n=0$ part survives because of the axial anomaly in the $y$-direction.

The effect is related to the winding number of the gap in Eq. (22) [21,22],

$$
\begin{aligned}
\int \frac{d^{2} p}{16 \pi} \operatorname{tr}[\hat{\mathbf{g}} \cdot(\nabla \hat{\mathbf{g}} \times \nabla \hat{\mathbf{g}})] & =\operatorname{sgn}\left(\varepsilon_{x} \varepsilon_{y}\right), \\
\mathbf{g}(\mathbf{p}) & =\left(\begin{array}{c}
\operatorname{Re}\left[\Delta(\mathbf{p})\left(-i \sigma_{2}\right)\right] \\
-\operatorname{Im}\left[\Delta(\mathbf{p})\left(-i \sigma_{2}\right)\right] \\
\left(\mathbf{p}^{2} / 2 m\right)-\epsilon_{\mathrm{F}}
\end{array}\right),
\end{aligned}
$$

where $\nabla=\partial / \partial \mathbf{p}$. This suggests that these effects occur even if $\varepsilon_{x}$ and/or $\varepsilon_{y}$ are infinitesimally small, and that these effects come from the P- and T-violation of the gap.

The accumulated mass density exists in the bulk region of the superfluid. In the superconductors, the Meissner effect occurs and the magnetic field cannot penetrate into the bulk, therefore the accumulated charge density would exist near the edge [26] of the superconductors and also around the vortex.

Let us roughly estimate the charge distribution around the vortex under uniaxial pressure. We make an approximation that the magnetic field is linearly decreased around the vortex core and vanishes at $r=\lambda_{\mathrm{L}}$, where $r$ is the distance from the core and $\lambda_{\mathrm{L}}$ is the London penetration depth; therefore, the gradient of the magnetic field is $-H_{c 2} / \lambda_{\mathrm{L}}$, where $H_{c 2}$ is an upper critical field along the $c$-axis. We use the parameters appropriate for $\mathrm{Sr}_{2} \mathrm{RuO}_{4}, H_{c 2}=0.075$ Tesla, $\lambda_{\mathrm{L}}=1800 \AA$ and $v_{\mathrm{F}}=10^{6} \mathrm{~cm} / \mathrm{s}$. The induced charge density around the vortex core obtained from Eq.(14) is of the order of $-10^{-8} e$ per $1 \AA^{2}$, when $\operatorname{sgn}\left(\varepsilon_{x} \varepsilon_{y}\right)=+1$. For the charge neutrality, there should be a plus charge $\pi \times(1800[\AA])^{2}$ $\times 10^{-8} e\left[\AA^{-2}\right]=10^{-1} e$ at the core. The core charge might extend in the region $r<\xi$ for the stability. Here $\xi=\pi v_{\mathrm{F}} / \Delta$ is the coherent length. Further (self-consistent) calculation is required to obtain more accurate results.

In summary, we have analyzed the $2 \mathrm{D}$ chiral $p$-wave superconductor near the second superconducting transition point [2,3] at sufficiently low temperature. Such a situation is described by a quasi-1D model in 2D, which can be mapped onto the 2D Dirac QED. 
Corresponding to the QHE in 2D Dirac theory [10 13], we show that the charge accumulation occurs under an inhomogeneous magnetic field as an effect of the axial anomaly in 1D. The effect is related to the winding number of the gap [21,22], and could contribute to the vortex core charge.

Recently, the vortex in chiral superconductors has been discussed [27], and such a vortex has a fractional charge and a fractional angular momentum. Interesting phenomena related to these fractional quantum numbers and the present effects are expected to occur around the vortex core.

An anisotropic chiral $p$-wave state, which has the $\mathbf{d}(\mathbf{k})=\hat{\mathbf{z}}\left(\sin k_{x}+i \sin k_{y}\right)$ order parameter, is proposed [28]. This state has four tiny gap points, therefore our argument would be applicable to quasiparticle excitations in the low temperature region of such a state [29].

The author is grateful to K. Ishikawa, R. Joynt, N. Maeda, K. Shizuya and M. Sigrist for useful discussions and encouragement. 


\section{REFERENCES}

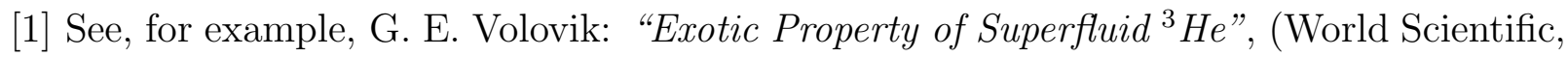
Singapore, 1992).

[2] G. M. Luke, et. al.: Nature 394 (1998) 558.

[3] M. Sigrist, R. Joynt and T. M. Rice: Europhys. Lett. 3 (1987) 629.

[4] S. L. Adler: Phys. Rev. 177 (1969) 2426.

[5] W. Bardeen: Phys. Rev. 184 (1969) 1848.

[6] J. S. Bell and R. Jackiw: Nuovo Cim. 60A (1969) 47.

[7] K. Fujikawa: Phys. Rev. Lett. 42 (1979) 1195.

[8] K. Fujikawa: Phys. Rev. Lett. 44 (1980) 1733.

[9] M. Atiyah and I. Singer: Ann. Math. 87 (1968) 484.

[10] A. Niemi and G. Semenoff: Phys. Rev. Lett. 51 (1983) 2077.

[11] A. Redlich: Phys. Rev. Lett. 52 (1984) 18.

[12] K. Ishikawa: Phys. Rev. Lett. 53 (1984) 1615.

[13] K. Ishikawa: Phys. Rev. D 31 (1985) 1432.

[14] K. Ishikawa and T. Matsuyama: Z. Phys. C 33 (1986) 41.

[15] K. Ishikawa and T. Matsuyama: Nucl. Phys. B280 (1987) 523.

[16] N. Imai, et. al.: Phys. Rev. B42 (1990) 10610.

[17] G. E. Volovik: Sov. Phys. JETP 65 (1987) 1193.

[18] M. Stone and F. Gaitan: Annals of Phys. 178 (1987) 89.

[19] B. Sakita and K. Shizuya: Phys. Rev. B, 42 (1990) 5586. 
[20] N. Nagaosa and M. Oshikawa: J. Phys. Soc. Jpn. 652241 (1996).

[21] G.E. Volovik: Sov. Phys. JETP 67 (1988) 1804.

[22] J. Goryo and K. Ishikawa: Phys. Lett. A 260 (1999) 294.

[23] D. F. Agterberg: Phys. Rev. Lett. 80 (1998) 5184.

[24] The Dirac Lagrangian is also obtained around tiny gap points of the $d_{x^{2}-y^{2}}+i \varepsilon d_{x y}$ superconductor with small $\varepsilon$. See, T. Senthil, J. B. Marston and Matthew P. A. Fisher: cond-mat/9902062. There are other applications of the Dirac Lagrangian to 2D condensed matter systems. See, for example, ref. 12, 13 and F. D. M. Haldane: Phys. Rev. Lett. 61 (1988) 2015; M. Oshikawa: Phys. Rev. B 50 (1994) 17357.

[25] R. Jackiw: "Lectures in Les Houches summer school, Session XL" (1983) 221.

[26] Note that the axial anomaly discussed here is in the $y$-direction, which is perpendicular to the edge, therefore it is different from that of the chiral edge mode discussed in, for example, X. G. Wen: Phys. Rev. Lett 64 (1990) 2206; S. Iso, D. Karabari and B. Sakita: Nucl. Phys. B 388 (1992) 700; N. Maeda: Phys. Lett. B 376 (1996) 142; N. Read and D. Green: Phys. Rev. B 61 (2000) 10267.

[27] J. Goryo: Phys. Rev. B 61 (2000) 4222.

[28] K. Miyake and O. Narikiyo: Phys. Rev. Lett. 83 (1999) 1423.

[29] J. Goryo, et. al.: in preparation. 


\section{FIGURES}

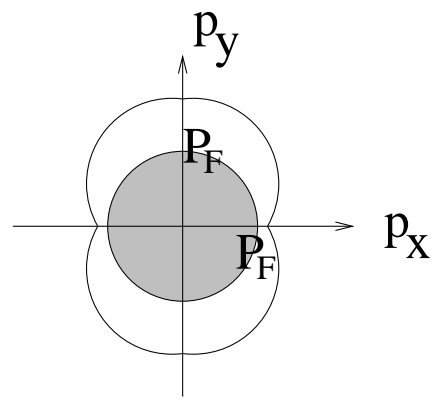

FIG. 1. The Fermi sea in the normal state and the momentum dependence of the gap functions for $\mathrm{Sr}_{2} \mathrm{RuO}_{4}$ near the second superconducting phase transition point. The shadows show the Fermi sea, and the distance between outer lines and inner lines shows the magnitude of the gap $\left|\Delta / p_{\mathrm{F}}\right|^{2}\left(\varepsilon_{x}^{2} p_{x}^{2}+\varepsilon_{y}^{2} p_{y}^{2}\right)$. 